\title{
A novel mechanical thrombectomy device based on complex longitudinal-torsional vibration
}

\author{
Minoru MORITA*, Jingjing YANG**, Yasuyoshi FUJIOKA* and Zhongwei JIANG* \\ * Graduate School of Science and Engineering, Yamaguchi University, \\ Ube, Yamaguchi 755-8611, Japan \\ E-mail:mmorita@yamaguchi-u.ac.jp \\ ${ }^{* *}$ Kunming University of Science and Technology,No.727 South Jingming Road, \\ Chenggong District, 650500, Kunming, China
}

Received: 30 March 2018; Revised: 9 July 2018; Accepted: 23 July 2018

\begin{abstract}
Recently medical treatment device using a complex vibration is developed, it has been designed for the improvement of the conversion efficiency. However, few reports are available on the vibration transducers as used in small blood vessel. In this paper, the new type longitudinal-torsional complex vibration transducer was designed. The principle of vibration conversion from longitudinal vibration to longitudinal-torsional complex vibration was described in detail. In order to obtain the most effective performance, the structure parameters of the vibrator involving the mode conversion were mentioned. The important parameters to achieve the highest conversion efficiency from longitudinal vibration to torsional vibration were investigated by using the finite element method analysis models. The angled-multilayered converter was designed, and the important parameters as layers offset angle and lead angle of twist structure were discovered. The most effective parameters were obtained for the offset angle was 10 degree and the lead angle was 56-59 degree. It was confirmed that the vibration conversion efficiency was improved by making a hole for the suction into the device.
\end{abstract}

Keywords : Structural design, Optimal design, Vibration convertor, Longitudinal-torsional complex vibration, FEM

\section{Introduction}

Thrombus, also known as blood clot, formed by blood coagulation, which blocks blood flow and leads to several serious medical problems such as heart attack and stroke. In the countries especially with high income, the ischemic heart disease and stroke induced by clot occlusion are always the three-leading members of the disease causes (Lozano et al., 2012, GBD 2013, Bennett et al., 2014). Due to the blocking-up of the supply of oxygen and glucose, the blocked blood vessel may lead to tissue injury or even death if it is not re-opened timely. The primary clinical treatment for dissolving thrombus utilizes intravenous thrombolysis agent with tissue plasminogen activator (t-PA) (The National Institute of Neurological Disorders and Stroke rt-PA Stroke Study Group, 1995). However, owing to the limited time window within 3 or 4.5 hours, and inadequate public awareness for seeking medical assistance quickly after the symptom onset, many patients have missed the opportunity of thrombolytic therapy (Davis and Donnan, 2009, Clark and Madden, 2009, Demers et al., 2012, Park et al., 2014). It is estimated that only 3\%-10\% of patients are eligible for receiving t-PA (Tenser et al., 2011). Moreover, a large amount of t-PA brings high risk of hemorrhage. Therefore, the successful treatment of ischemic stroke and heart disease crucially depends on time, short recanalization time is urgently desired after the symptom onset (Pereira et al., 2015).

Over the past decades, many mechanical thrombectomy devices for rapidly reopen occlusive blood vessel were developed to acute thrombosis. These devices can be used beyond the time window of thrombolytics therapy. Moreover, they have higher recanalization rate and favorable clinical outcomes (Fanous et al., 2016). The existing mechanical thrombectomy devices, as known as Merci Retriever (Stryker, Kalamazoo, MI, USA), Penumbra (Penumbra Inc.,Alameda, CA, USA) and Solitaire Flow Restoration (v3/Covidien, Irvine, CA, USA) were approved by United States 
Food and Drug Administration (FDA) for treating ischemic stroke. Since the advantages of short time, high rate of recanalization and high rate of clinical outcomes, these devices were considered as the most efficient device for vessel recanalization. However, the complication of vascular damage was found when the unfolded stent threaded through the blood vessel (Arai et al., 2016). Furthermore, the present devices are usually used in relatively larger blood vessel with diameter ranges from $2 \mathrm{~mm}$ to $5 \mathrm{~mm}$ (Lutsep, 2015). Therefore, a new device which can dissolve or remove the blood clot in relatively small blood vessel timely without vascular intimal damage is desired.

An advanced method by stirring the blood clot based on mechanical vibration with injection of a few thrombolytic agent into the clot to accelerate the clot dissolution was proposed (Jiang et al., 2003, Ajoudanian et al., 2011a, 2011b, 2013, Morita et al., 2013). In this therapy, a micro-actuator, composed with a shear-actuated cantilever beam which can generate transverse vibration at the beam tip for accelerating clot dissolution was designed. Since the stirring function, clot was fragmented into small pieces and mix with agent fully, so that the clot could be dissolved in a short time. Moreover, due to the use of tiny amount of agent, the risk of hemorrhage is low. In addition, no damage will be caused because there is no touch between the stirrer and vascular intimal. However, since the tip deflection of beam depends on the beam's length, it is hard to obtain transversal deflection with enough power in slender-narrow blood vessel. Furthermore, due to the long distance from the surgical incision to the site of occlusion, the power transmission of the actuator is another significant challenge. To overcome the above problems, we proposed a new device with complex longitudinal-torsional vibration for smashing and accelerating thrombus dissolution in narrow blood vessel. The device consists of a long-hollowing wire with an angled-multilayer converter at one side and a transducer at the other side. The longitudinal force, produced by transducer outside of body, is delivered to the clot's site and then partly converted into torsional vibration, so that generate longitudinal-torsional vibration at the wire tip for smashing clot. Finally, the clot fragments are aspirated out of body through the center hollow. In this design, since the coaxial line of the converter and blood vessel, the converter can be made with small size, and the torsional vibration can be obtained easily in narrow blood vessel without contact damage. Further, a powerful transducer outside of body is used to support smashing force, so that there is no limitation on transducer size. As the major part of the device, the design of the converter for obtaining high conversion efficiency from the longitudinal vibration to torsional vibration is the important content in this research. The design concept of the converter was first explained in detail. The key design parameters of the converter have been proposed, and a series of finite element method (FEM) simulations were conducted to determine optimal parameters' values for obtaining highest conversion efficiency.

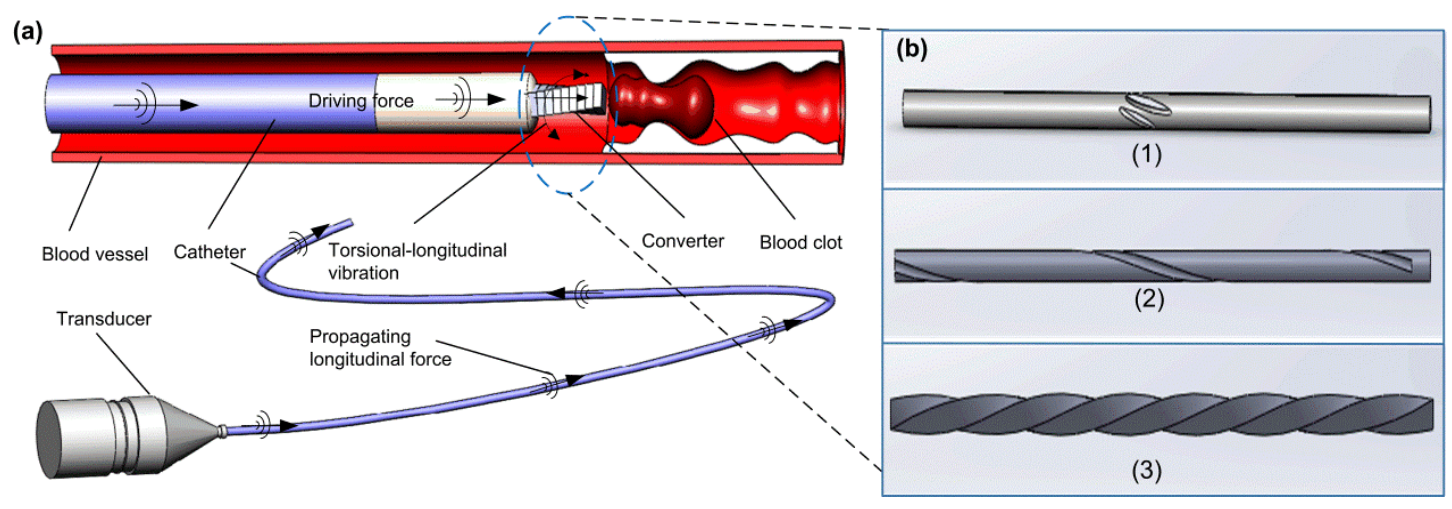

Fig. 1 The schematic of longitudinal-torsional device with (a) operation in blood vessel and (b) three types of converter. (1) Short spiral groove on the exterior surface of the bar, (2) Spiral groove on the exterior surface of the whole bar, (3) A twisted bar with rectangular cross section.

\section{Design concept}

In the endovascular treatment by using the proposed device, a micro-catheter is inserted through the femoral artery and delivered to the location of blood clot by a guide wire. Then, the guide wire is drawn out, and a micro-tube for agent injection is inserted within the micro-catheter and delivered to the blood clot with injection of t-PA. Moving the injecting tube out and inserting the smashing actuator into the catheter. After the actuator arriving at the occlusion site, excite the actuator to smash the blood clot, as well as aspirate the undissolved fragments out through the actuator's hollow. 
Schematically shown in figure 1 (a), the actuator consists of a long-hollowing wire with a torsional converter at one side and a powerful transducer at the other side. The longitudinal force excited by the transducer transmits through the wire and reaches to the converter, and then partly converts into torsional vibration due to the elastic force reflection in the special structure. Since the converter and blood vessel are coaxial, the diameter can be made with small size easily for slender vessel application. In this way, longitudinal-torsional vibration mode is excited to smash the blood clot.

However, the structure of converter has significant influence on the conversion efficiency. Due to the small size of blood vessel, the coaxial structure with the blood vessel of the converter was preferred. Three typical types of designs for converting longitudinal vibration to torsional vibration coaxially were taken into account, as shown in figure.1 (b). The type 1 (figure 1 b-1,2) is designed with a spiral groove on the exterior surface of the bar (Boukhny et al., 2001, 2006), and the type 2 (figure 1 b-3) is a twisted bar with rectangular cross section (Friend et al., 2005, Miura and Asami, 2013). It should be noted that, the diameter of the converter should be very small due to the small profile of blood vessel. The type 1 with spiral groove and type 2 with twisted structure were selected to be considered for make a complex vibration.

In order to manufacture the structure of type 2, a solid cuboid needs to be twisted by torque. A FEM model, as the shown as figure 2, was built to analyze the stress distribution by finite element analysis software of ANSYS. A cantilever beam with length of $43 \mathrm{~mm}$ and width of $1.3 \mathrm{~mm}$ was built, and a moment of couple with value of $0.1 \mathrm{Nm}$ was loaded on its free tip. The material of the bar was stainless steel (SUS304) with yield stress of $205 \mathrm{MPa}$ and tensile stress of 520MPa. As the simulation result shown in figure 2 (Twisted type), it's obvious that the stress values at the center and free tip were 346.2 $\mathrm{MPa}$ and 889.9 MPa respectively which exceeded the yield stress and tensile stress of material. However, the number of turn was only 0.125 . Therefore, the manufacture of the twisted converter without metal fracture is difficult. A new idea to solve the manufacturing problem of type 2 is to cut serious angled-square blocks on the surface of cylindrical bar, as shown as figure 3. In this way, the bar doesn't need to be twisted with no risk of broken. Hereinafter, this new idea device called angled-multilayered type.

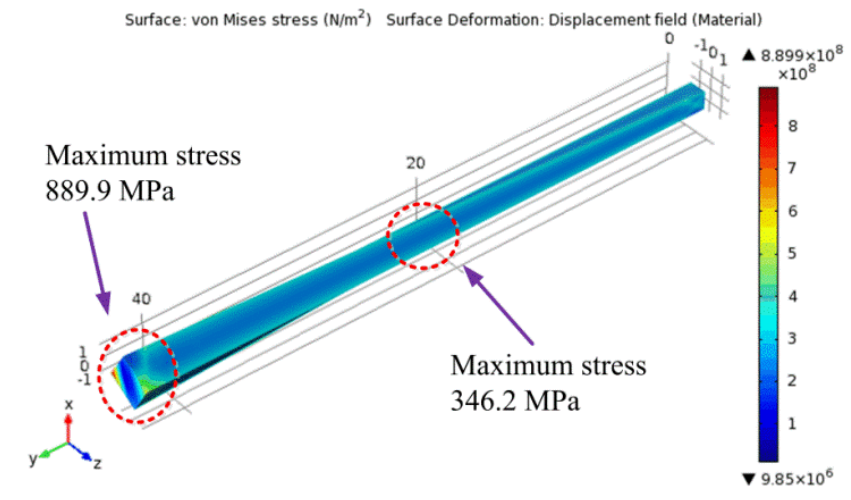

Fig. 2 FEM stress distribution of a twist bar. The maximum stress values was $889.9 \mathrm{MPa}$ which exceeded the tensile stress of material despite the rotation amount being 0.125 .

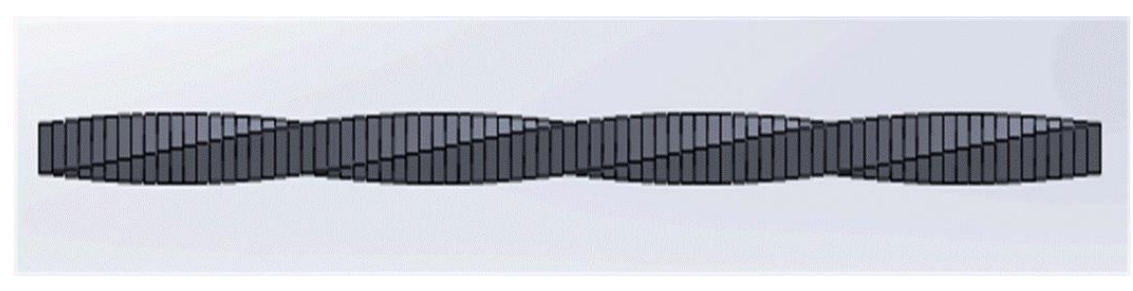

Fig. 3 Longitudinal-torsional converter with angled-multilayered beam

The profile of the converter crucially affects the conversion efficiency from longitudinal vibration to torsional vibration. Aiming for evaluating the conversion efficiency, three FEM models with profiles of type 1 (figure 1, b-2), type 2 (figure $1 \mathrm{~b}-3$ ) and new design respectively were built. Considering the small size of blood vessel, the diameter of the model of type 1 was set up with $1.3 \mathrm{~mm}$, as same as the side length of the square block of type 2 and angled-multilayered structure. All of the lengths of models were set up with $43 \mathrm{~mm}$ and number of turns was 1 . For type 1, the radius of the surface groove was $0.3 \mathrm{~mm}$. For angled-multilayered structure, the offset angle between adjacent blocks was $10^{\circ}$, so that 
the bar consisted of 37 blocks with width of $1.6 \mathrm{~mm}$. The material of both these three models in following simulation was stainless steel (SUS 304), and its material characteristics of Young's Modulus, Poisson's Ratio and Density were 197 $\mathrm{GPa}, 0.3$ and $8000 \mathrm{Kg} / \mathrm{m} 3$ respectively. The left end of the bar was loaded by a sinusoidal force with amplitude of $1 \mathrm{~N}$, and other boundary conditions were set for free.

The frequency response analysis results are exhibited in Figure 4. It is obvious that with the same boundary condition and size, the angled-multilayered structure decreased the conversion efficiency compared with twist structure. However, the new design generated bigger torsional angle than the convertor with spiral groove. Considering that the new model doesn't need to twist processing, the angled-multilayered converter is judged as the most effective and it was adapted for the vibration convertor of this paper. The converter design is discussed in detail in following.

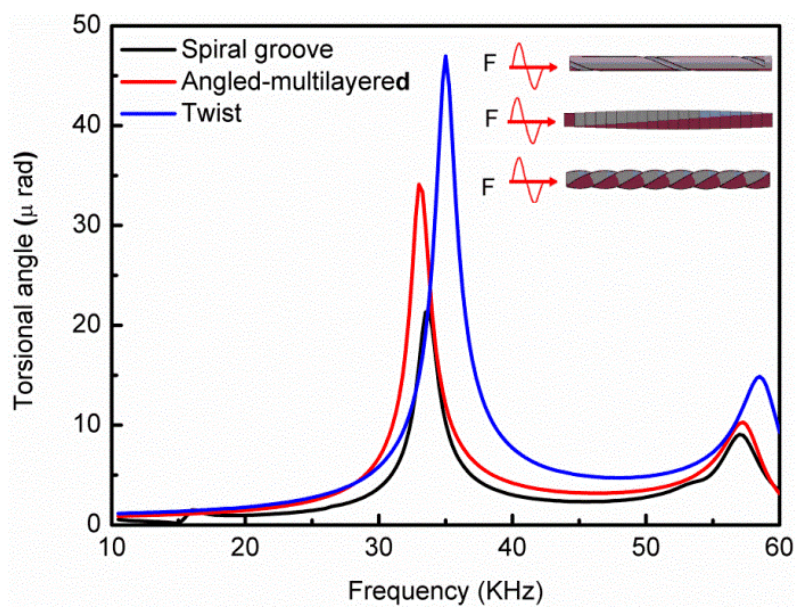

Fig. 4 Tip torsional angle of different converters at the resonant frequencies. Here, Spiral groove is model of figure $1 \mathrm{~b}-2$, Twist is model of figure 1 b-3 and Angle-multilayered is our new idea (figure 3).

\section{Structure design and modeling}

Considering the angled-multilayered convertor needs to be inserted in blood vessel to smash blood clot in narrow space, its small size will affect the performance of the motion conversion, as well as affect the smashing effect. Therefore, to determine the converter structure with the higher efficiency, the key design parameters should be analyzed. As the theoretical model shown in figure 5, considering a bar with left end is fixed and twisted by a torque $T$, the small torsional angle can be described as

$$
\theta_{t}=\frac{T l}{G I_{p}}
$$

Where is the torsional angel, $T$ is the applied torque, $l$ is the length of bar, $G I_{p}$ is the torsional rigidity. With the angled-multilayered structure of the bar, as figure 6 shows, the length of bar should be written in the form

$$
l=N d=\left(\frac{2 n \pi}{\theta}+1\right) d
$$

Here, $N$ is the number of block, $n$ is the number of turns, $\theta$ is the offset angle, $w$ is the width of the block and $d$ is the thickness of the blocks respectively. In addition, the lead angle which is an important parameter of the twisted bar can be described as the function of $\theta, t$ and $w$

$$
\theta_{L}=\arctan \left(\frac{N d}{2 \pi R}\right)=\arctan \frac{(2 n \pi+\theta) d}{\sqrt{2} \pi \theta_{v}}
$$




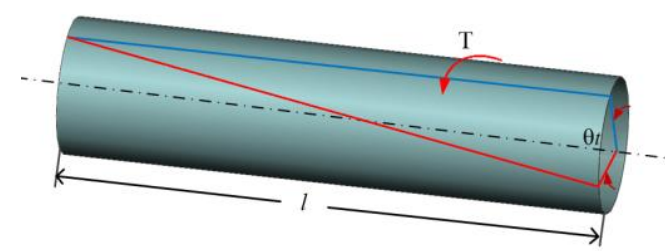

Fig. 5 Torsional motion of a bar with applied torque. A bar with left end is fixed and twisted by a torque $T$. $\theta_{t}$ is torsional angle.

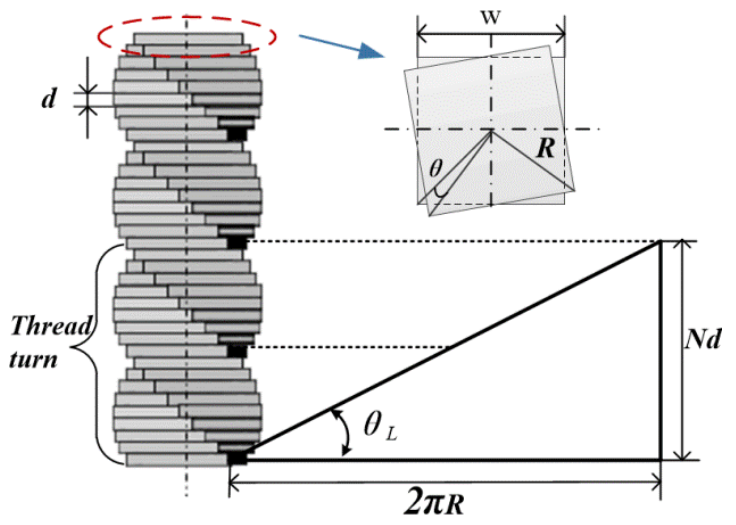

Fig. 6 Structure details for calculating lead angle

Therefore, it's obviously that $\theta_{t}$ is the function of $\theta, n, \theta_{L}, w$ and $t$, which can be described as

$$
\theta_{t}=\frac{\sqrt{2} \pi w T \tan \theta_{L}(2 n \pi+\theta)}{G I_{p} N \theta}
$$

Based on the above equations, three typical parameters of the converter including offset angle, number of turns and lead angle which relate with other parameters closely have been selected to analyze the performance.

\subsection{Offset angle}

To analyze the relationship between the converted torsional angle and offset angle of blocks, three FEM models with offset angles 10 degree, 20 degree and 30 degree respectively were built by software ANSYS. The left end of the convertor was loaded by sinusoidal force with amplitude of $1 \mathrm{~N}$, and the sweep frequency of the force was ranged from $30 \mathrm{KHz}$ to $60 \mathrm{KHz}$. The material of the convertor was stainless steel (SUS304), and the length and number of turns of the three bars were $43 \mathrm{~mm}$ and 1 respectively. All of the boundary conditions were set for free. The other parameters of the actuator are listed in table 1.

Table 1 Parameters of the converter.

\begin{tabular}{lccc}
\hline Offset angle " $\theta$ " [degree] & 10 & 20 & 30 \\
\hline Width of block " $w$ " $[\mathrm{mm}]$ & 1.3 & 1.3 & 1.3 \\
Number of block " $N$ " & 37 & 19 & 13 \\
Thickness of block " $d$ " $[\mathrm{mm}]$ & 1.47 & 2.26 & 3.31 \\
\hline
\end{tabular}

As the frequency response analysis shown in figure 7, the torsional angle at the tip of the bar with offset angle of 10 degree was biggest, while the bar with offset angle of 30 degree was smallest. In other words, as the same input force is loaded on the actuator, the smaller offset angle of the block can be expected, the bigger torsional vibration can be obtained. However, considering the practical difficulty of manufacture, the smaller offset angle less than 10 degree is difficult to cut on a thin bar with diameter under $2 \mathrm{~mm}$. Therefore, the offset angle with 10 degree was selected. 


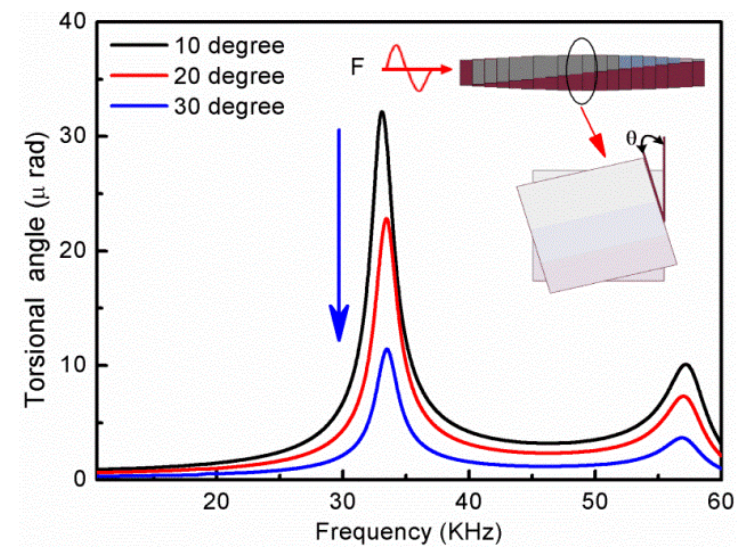

Fig. 7 Tip torsional angle of converters with different offset angles

\subsection{Turn number}

The turn number is another important parameter which may has crucial influence on the motion conversion efficiency. In order to evaluate the torsional vibration with different turn numbers, 10 models with turn number from 1 to 10 were built and simulated. All of the models have the same length of $43 \mathrm{~mm}$, as well as with the same offset angle of 10 degree and same side length of $1.3 \mathrm{~mm}$. The left end of the bar was loaded by a longitudinal sinusoidal force of $1 \mathrm{~N}$ and the other boundary conditions were set for free. The other parameters were listed in table 2 .

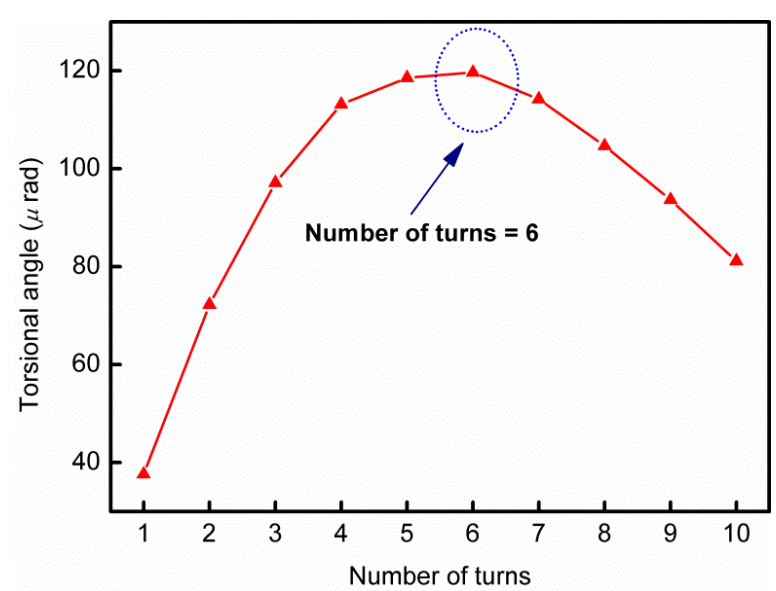

Fig. 8 Simulation results of torsional angle with different turns
Table 2 Parameters of the converter with different turn numbers.

\begin{tabular}{lcc}
\hline $\mathrm{n}$ & $\mathrm{N}$ & $\mathrm{t}[\mathrm{mm}]$ \\
\hline 1 & 37 & 1.16 \\
2 & 73 & 0.59 \\
3 & 109 & 0.39 \\
4 & 145 & 0.30 \\
5 & 181 & 0.24 \\
6 & 217 & 0.20 \\
7 & 253 & 0.17 \\
8 & 289 & 0.15 \\
9 & 325 & 0.13 \\
10 & 361 & 0.12 \\
\hline
\end{tabular}

The torsional angle of the right tip were measured and shown in figure 8 . The torsional angle has biggest value of $117 \mu$ rad with turn number of 6 . This meant the highest conversion efficiency can be obtained under 6 turns in this model. In the above models, the width of block and block number changed along with the turn number and bar's length. These parameters may also affect the conversion efficiency. Aiming for evaluating the influence of these parameters on conversion efficiency, another 8 FEM models with different parameters were performed for confirming the influence. The model parameters and simulation results were shown in table 3.

As the simulation results shown in table 3 , the turn number of converter with biggest torsional angle under different length and width were random ranging from 2 to 8 . With the same length of converter, such as $\mathrm{L}=43 \mathrm{~mm}$ and $\mathrm{L}=30 \mathrm{~mm}$, as the increase of block's width, the max torsional angle and corresponding turn number will decrease. In addition, under the same size of width, the bigger output torsional angle can be obtained along with the increase of length and turn number. All of the results demonstrated that the conversion efficiency of the converter with angled-multilayered structure is not determined by the turn number only. Therefore, the optimal turn number is not a certain value in converter's design. 
Morita, Yang, Fujioka and Jiang,

Journal of Advanced Mechanical Design, Systems, and Manufacturing, Vol.12, No.4 (2018)

Table 3 Parameters of converter with different length and thickness.

\begin{tabular}{lcccc}
\hline Parameter & $\begin{array}{c}\text { Length } \\
{[\mathrm{mm}]}\end{array}$ & Width[mm] & Turn number & $\begin{array}{c}\text { Max torsional } \\
\text { angle }[u \text { rad }]\end{array}$ \\
\hline L43_W1.3 & 43 & 1.3 & 6 & 120 \\
L43_W2.0 & 43 & 2.0 & 4 & 33 \\
L43_W2.6 & 43 & 2.6 & 3 & 15 \\
L30_W1.3 & 30 & 1.3 & 4 & 76 \\
L30_W2.0 & 30 & 2.0 & 3 & 20 \\
L30_W2.6 & 30 & 2.6 & 2 & 9.6 \\
L60_W1.3 & 60 & 1.3 & 8 & 160 \\
L86_W2.5 & 86 & 2.5 & 6 & 29 \\
\hline
\end{tabular}

\subsection{Lead angle}

The lead angle is another important parameter needs to be considered for improving conversion efficiency. In order to evaluate the influence of lead angle on motion conversion, three models of the above 8 models have been selected to simulate. These three models have the same block width of $1.3 \mathrm{~mm}$ but different lengths of $30 \mathrm{~mm}, 43 \mathrm{~mm}$ and $60 \mathrm{~mm}$.

The calculated results between lead angle and torsional angle were shown in figure 9. According to the curves trend, under the same conditions, the converted torsional angle increased along with the increase of convertor length. It demonstrated that the length of the convertor can magnify the torsional angle at the free tip so that longer convertor can obtain bigger torsional angle. Note that, the lead angles in which the biggest torsional angles were obtained have been marked and covered by a blue band. It is obvious that the preferred angles for obtaining highest motion conversion concentrated on the lead angle between 56 degree and 59 degree. Therefore, the design of ideal lead angle can be determined with these proper values.

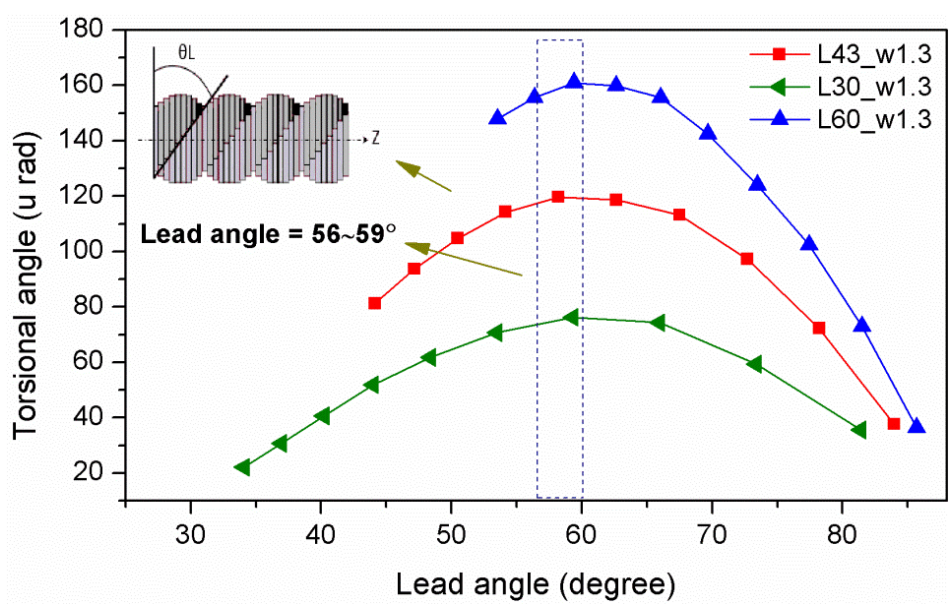

Fig. 9 Lead angles of converters with different parameters. The preferred angles for obtaining highest motion conversion was concentrated on the lead angle between 56 degree and 59 degree.

\section{Evaluation and discussion}

The optimal parameters of the angled-multilayered structure for obtaining high conversional efficiency have been determined. Considering that the device should have the aspirating function to aspirate the clot fragments outside of body, the conversion efficiency of the converter with different sizes of center hollow should be finally taken into account. In common sense, the center hollow will decrease the energy transmission due to the removal of solid material. However, as it is known to all, the center hollow will also decrease the stiffness of bar, which makes the bar easy to rotate to obtain relatively big torsional angle and eccentric force. The eccentric force is the key to generate transversal vibration which is used to impact and smash the blood clot in vessel's radial direction, as well as the longitudinal vibration impacts the 
clot in the axial direction of blood vessel. Therefore, the transversal vibration amplitude and longitudinal vibration amplitude are the key elements to characterize the final conversion effect.

To evaluate the converter's conversion effect with different center hollows, the models of converter with arbitrary hollow radius as $0.0 \mathrm{~mm}, 0.2 \mathrm{~mm}, 0.4 \mathrm{~mm}$ and $0.5 \mathrm{~mm}$ and same length of $43 \mathrm{~mm}$ were simulated respectively. Other parameters including the offset angle, turn number and lead angle were set up with the optimized values as 10 degree, 6 turns and 59 degree. The left end of the convertor was loaded by a longitudinal-sinusoidal force with amplitude of $1 \mathrm{~N}$, and the other boundary conditions were set for free.

The schematic of the hollowing converter was shown in figure 10. The transversal vibration amplitude, longitudinal vibration amplitude and the amplitude ratio of the bar's right tip with hollow radius of $0.0 \mathrm{~mm}, 0.2 \mathrm{~mm}, 0.4 \mathrm{~mm}$ and 0.5 $\mathrm{mm}$ are shown in figure 11. Obviously, the torsional amplitude, longitudinal amplitude and amplitude ratio increased along with the increase of hollow radius. It demonstrated that the removal of material didn't reduce the energy transmission but enhanced the motion in both longitudinal and transversal directions. Moreover, according to the increasing curve, the center hollowing convertor has greater influence on the transversal deflection and torsional vibration which are the most important design goals of the converter. Therefore, in consideration of the whole size of device and blood vessel, the hollow should be made as big as possible due to the good performance in the simulations. Simultaneously, the above designed converter can generate effective vibration in small blood vessel for accelerating thrombus dissolution.

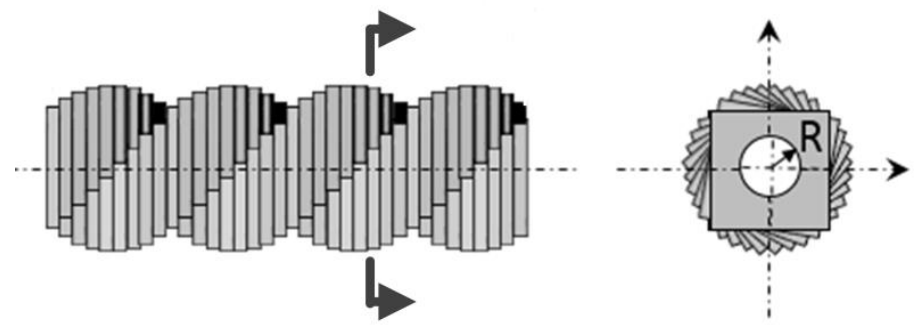

Fig. 10 Converter with center hollow

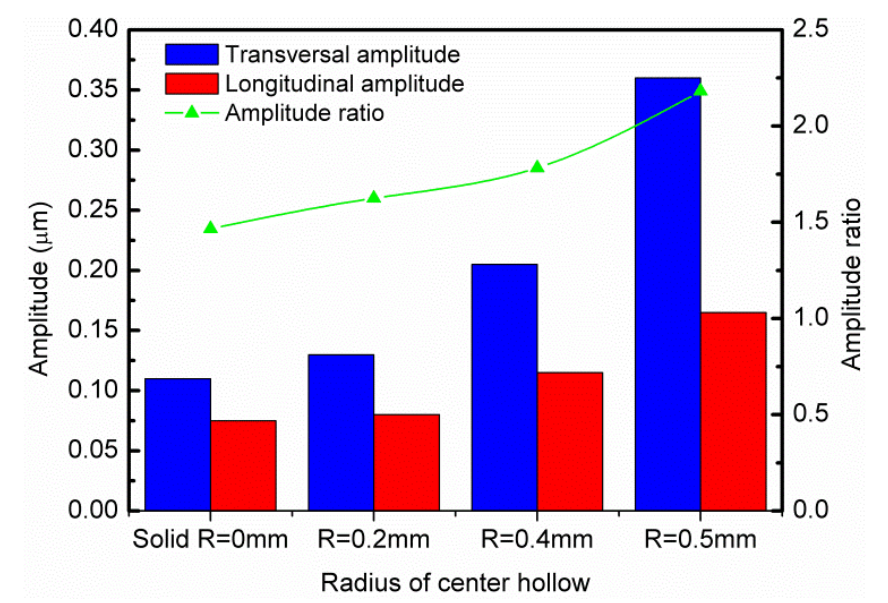

Fig. 11 Tip amplitude of converter with different sizes of center hollows

\section{Conclusion}

In this paper, a new device which can supply efficient longitudinal-torsional vibration in small blood vessel for accelerating thrombus dissolution was designed. The angled-multilayered converter was designed, and the important parameters as layers offset angle and lead angle of twist structure were discovered. The effective design parameters of the converter have been released, and the optimal parameters have been determined based on the analysis of several FEM models. The most effective parameters were obtained for the offset angle was 10 degree and the lead angle was 56-59 degree. It was confirmed that the vibration conversion efficiency was improved by making a hole for the suction into the device. The simulation results illustrated that with the optimized design parameters, the converter can supply effective compound longitudinal-torsional vibration. 


\section{References}

Ajoudanian, M., Jiang, Z. W. and Morita, M., Study on a New Type Micro-stirrer Excited by Longitudinal Elastic Wave for Thrombus Dissolution, Int. J. Appl. Electromagn. Mech. 37 (2011a), pp.67-77.

Ajoudanian, M., Jiang, Z. W. and Morita, M., Design of a Novel Type Micro-Stirrer Excited by Longitudinal Elastic Wave for Thrombus Dissolution, J. Biomech. Sci. Eng. 6(4) (2011b), pp.262-269.

Ajoudanian, M., Jiang, Z. W. and Morita, Structural Analysis and Design of Micro-stirrer Driven at a Requested Frequency for Thrombus Dissolution, Int. J. Appl. Electromagn. Mech. 41 (2013), pp.217-225.

Arai, D., Ishii, A., Chihara, H., Ikeda, H., Miyamoto, S., Histological examination of vascular damage caused by stent retriever thrombectomy devices, Journal of NeuroInterventional Surgery, Vol.8, Issue.10 (2016), pp.992-995.

Bennett, D. A., Krishnamurthi, R. V., Beamker-Collo, S., Forouzanfar, M. H., Naghavi, M., Connor, M., Lawes, C. M. M., Moran, A. E., Anderson, L. M., Roth, G. A., Mensah, G. A. Ezzati, M., Murray, C. J.L., Feigin, V. L., The Global Burden of Ischemic Stroke Findings of the GBD 2010 Study, Glob. Heart 9 (2014), pp.107-112.

Boukhny, M., L. Beach, Torsional Ultrasound Tip, United States Patent disclosure US 2001/0011176 A1. (2001).

Boukhny, M., Chon J. Y., Salehi, A., Ultrasound Handpiece, United States Patent disclosure US 2006/0041220 A1. (2006).

Clark, W. M., Madden, K. P., Keep the Three Hour TPA Window: The Lost Study of Atlantis, Journal of Stroke and Cerebrovascular 18(1) (2009), pp.78-79.

Davis, S. M., Donnan, G.A., 4.5 Hours: The New Time Window for Tissue Plasminogen Activator in Stroke, Stroke 40 (2009), pp.2266-2267.

Demers, G., Meurer, W. J., Shih, R., Rosenbaum, S., Vilke, G. M., Tissue Plasminogen Activator and Stroke: Review of the Literature for the Clinician, J. Emerg. Med. 43(6) (2012), pp.1149-1154.

Fanous, A. A., Siddiqui, A. H., Mechanical thrombectomy: Stent retrievers vs. aspiration catheters, Cor et Vasa 58(2) (2016), pp.193-203.

Friend, J., Nakamura, K., Ueha, S., Vertical - torsional vibration transducer and tortional vibrator., Japanese patent disclosure JP2005-08121 (2005). (in Japanese).

GBD 2013 Mortality and Causes of Death Collaborators, Global, Regional, and National Age-sex Specific All-cause and Cause-specific Mortality for 240 Causes of Death, 1990-2013: A Systematic Analysis for The Global Burden of Disease Study 2013, Lancet 385 (9963) (2015), pp.117-171.

Jiang, Z. W., Liu, Z. H., Kato, S., Suzuki, M., Fundamental Study on a Novel Method for Dissolution of Cerebral Thrombus by Micro Piezo-stirrer, in: Proceedings of Joint Conference on Micromechatronics for Information and Precision Equipment, (2003), pp.379-380.

Lozano, R., Naghavi, M., Foreman, K., Lim, S., Shibuya, K., Aboyans, V., et al., Global and Regional Mortality from 235 Causes of Death for 20 Age Groups in 1990 and 2010: A Systematic Analysis for the Global Burden of Disease Study 2010, Lancet 380 (9859) (2012), pp.2095-2128.

Lutsep, H. L., Mechanical Thrombolysis in Acute Stroke (online), http://emedicine.medscape.com/article/1163240overview\#a1, (2015), (accessed on 8 May, 2017).

Miura, H., Asami, T., Ultrasonic complex Vibrator, Japanese patent disclosure JP2013-111508 (2013). (in Japanese).

Morita, M., Jiang, Z. W., Yamamoto, K., Suzuki, M., Design and Development of Hinge-type Microstirrer for Thrombus Dissolution, Int. J. Appl. Electromagn. Mech. 41 (2013), pp.87-96.

Park, T. H., Lee, J. S., Park, S., Ko, Y., Lee, S. J., Lee, K. B., et al., Safety and Efficacy of Intravenous Recombinant Tissue Plasminogen Activator Administered in the 3 to 4.5 Hour Window in Korea, Journal of Stroke and Cerebrovascular Diseases 23(7) (2014), pp.1805-1812.

Pereira, V.M., Yilmaz, H., Pellaton, A., Slater, L., Krings, T., Lovblad, K., Current Status of Mechanical Thrombectomy for Acute Stroke Treatment, Journal of Neuroradiology 42(1) (2015), pp.12-20.

Tenser, M. S., Amar, A. P., Mack, W. J., Mechanical Thrombectomy for Acute Ischemic Stroke Using the MERCI Retriever and Penumbra Aspiration Systems, World Neurosurgery 76(6) (2011), pp.S16-S23.

The National Institute of Neurological Disorders and Stroke rt-PA Stroke Study Group, Tissue Plasminogen Activator for Acute Ischemic Stroke, N. Engl. J. Med. 333 (1995), pp.1581-1587. 